\title{
Descripción Anatómica de los Senos Paranasales de la Alpaca (Vicugna pacos)
}

\author{
Anatomical Description of the Sinus in the Alpaca (Vicugna pacos)
}
Rosse Zárate L. ${ }^{1}$, Miluska Navarrete Z..$^{1,4}$, Alberto Sato S. ${ }^{1}$, Diego Díaz C. ${ }^{2}$, Wilfredo Huanca L. $^{3}$

\section{Resumen}

El presente estudio tuvo por objetivo la descripción anatómica macroscópica de los senos paranasales de la alpaca. Se utilizaron 10 cabezas de alpacas adultas, donde la capa muscular se removió mediante la técnica de maceración. La descripción de los senos paranasales se hizo a través de cortes longitudinales empleándose la terminología recomendada por la Nómina Anatómica Veterinaria. Asimismo, se tomaron placas radiográficas con un medio de contraste a cuatro alpacas adultas vivas para determinar las relaciones con otras estructuras anatómicas. Los resultados demostraron que los senos frontal y maxilar se encuentran desarrollados y se comunican por el agujero frontomaxilar; el seno frontal se divide en medial y lateral, similar a los rumiantes menores; el seno maxilar es indiviso, similar a los rumiantes, pero se comunica con la cavidad nasal a través de la abertura nasomaxilar, similar al equino. No se evidenciaron los senos esfenoideos y etmoideos.

Palabras clave: alpaca, senos paranasales, anatomía

\section{Abstract}

The objective of the present study was to describe the macroscopic anatomy of the sinus in the alpaca. Ten skulls of adult alpacas were prepared by the maceration technique, and the description through longitudinal cuts was done using terminology recommended by the Nomina Anatomica Veterinaria. In addition, X-rays with contrast medium were

\footnotetext{
${ }^{1}$ Laboratorio de Anatomía Animal y Fauna Silvestre, ${ }^{2}$ Laboratorio de Farmacología y Toxicología

${ }^{3}$ Laboratorio de Reproducción Animal, Facultad de Medicina Veterinaria, Universidad Nacional Ma yor de San Marcos, Lima, Perú

${ }^{4}$ E-mail: mnavarretez@unmsm.edu.pe
}

Recibido: 5 de junio de 2014

Aceptado para publicación: 10 de marzo de 2015 
taken to four adult alpacas to determine the relationships of sinus with other anatomical structures. Results showed that the frontal and maxillary sinus were well developed and communicated through the frontomaxillary hole; the frontal sinus is divided into a medial and lateral portion as in small ruminants; the maxillary sinus is undivided and has a communication with the nasal cavity through the nasomaxillary opening as in the equine. The sphenoid and ethmoid sinuses were not evident.

Key words: alpaca, sinuses, anatomy

\section{INTRODUCCIÓN}

La crianza de camélidos sudamericanos (CSA) constituye una importante fuente de ingresos económicos para las empresas asociativas, pequeños productores y comunidades campesinas de la región andina. La zona altoandina del Perú se caracteriza por una alta incidencia de heladas y precaria disponibilidad de agua. En este medioambiente, a más de $3000 \mathrm{msnm}$, habitan las alpacas y las llamas debido a la ventaja que poseen de convertir con eficiencia la vegetación nativa en carne y fibras de alta calidad (Iñiguez y Alem, 1996).

El cráneo de alpaca se diferencia de los rumiantes (bovino, ovino, caprino) por la ausencia de cuernos. Presenta órbita ocular completa. El hueso incisivo presenta alveolo para el único diente incisivo de la arcada superior, mientras que el hueso maxilar presenta alveolo para el diente canino; siendo esto una diferencia con otros rumiantes que poseen rodete dentario en la arcada superior. En el límite de los huesos nasal, maxilar y frontal presenta un espacio o foramen que comunica la cavidad nasal con la cavidad ocular. La mandíbula presenta la sínfisis mandibular fusionada, así como el proceso subcondilar, característico de esta especie. Los huesos nasales son cortos (Navarrete y Sato, 2010).

La anatomía del aparato respiratorio de los camélidos sudamericanos no ha sido bien descrita, incluyendo la ausencia de información sobre los senos paranasales (Fowler,
2010). Autores como Sisson y Grossman (1993) y Koning y Liebich (2004) hacen referencia a los senos paranasales en caninos, felinos, bovinos, equinos y porcinos, en tanto que Fowler (2010) delimita los senos paranasales maxilar y frontal en un gráfico de cráneo de llama.

Los senos paranasales son divertículos de la cavidad nasal, que contienen aire y que están comunicados directa o indirectamente (Climent et al., 2005). Se localizan dentro de los huesos faciales del cráneo; y se cree que tienen como función neumatizar los huesos del cráneo aligerando el peso de la cabeza, así como zonas de intercambio de calor y lugares de secreción de mucus (Aspinall y O'Reilly, 2004).

El presente trabajo tuvo como objetivo la búsqueda de una visión anatómica y macroscópica de los senos paranasales.

\section{Materiales y Métodos}

\section{Material Biológico}

Se utilizaron 10 cabezas de alpacas adultas (5 hembras y 5 machos) de la raza Huacaya, aparentemente sanas, colectadas en el camal municipal de Nuñoa, provincia de Melgar, departamento de Puno, Perú. Las muestras fueron trasladadas al Laboratorio de Anatomía Animal de la Facultad de Medicina Veterinaria (FMV), Universidad Nacional Mayor de San Marcos (UNMSM), Lima. Además, se trabajó con cuatro alpacas adul- 
tas (2 hembras y 2 machos) vivas para la toma de placas radiográficas.

\section{Técnica de Maceración}

El retiro de los tejidos blandos adheridos a los huesos se realizó con la técnica de maceración en agua corriente por 30 días. Para ello se colocó el material de trabajo en recipientes con agua y luego se tapó herméticamente para que microorganismos saprófitos destruyan el material orgánico restante por espacio de 15 días. Posteriormente, se retiró el resto de material orgánico mediante lavado con agua a presión, proceso que se repitió en los 15 días siguientes.

La limpieza de los huesos se completó por medio de disección y sometiéndolos al efecto blanqueador de lejía en solución. Los huesos fueron secados por siete días y se procedió a la descripción de los huesos que conforman los senos paranasales, así como sus dimensiones, utilizando un vernier calibrado; y se determinaron las relaciones con otras estructuras anatómicas, siguiendo la terminología recomendada por la Nómina Anatómica Veterinaria (2012).

\section{Placas Radiográficas}

Se tomaron placas radiográficas de la cabeza a las cuatro alpacas Huacaya en la Clínica de Animales Menores de la FMVUNMSM, utilizando un equipo convencional de rayos X General Electric Mobile 225, de columna móvil, con capacidad máxima de $125 \mathrm{kVp}$ y de $200 \mathrm{~mA}$. Los animales fueron sedados con xilazina $0.2 \mathrm{mg} / \mathrm{kg}$ y ketamina $1.5 \mathrm{mg} / \mathrm{kg}$ (Fowler, 2010).

Se tomaron las dos primeras placas (latero-lateral y dorso-ventral) sin medio de contraste, como protocolo para tener una referencia. Luego, se inyectaron diferentes dosis de una sustancia de contraste (iopamidol), dado que se desconoce la dosis óptima para camélidos. Esta sustancia se inyectó por la fontanela lagrimal, característica de los camélidos sudamericanos, para visualizar mejor los senos paranasales en la placa radiográfica ( 2 y $3 \mathrm{ml}$ en la fontanela lagrimal en ambos lados en la primera y segunda alpaca, y 5 y $10 \mathrm{ml}$ en la fontanela lagrimal en lado izquierdo en la tercera y cuarta alpaca). Las placas radiográficas fueron utilizadas para confirmar la comunicación entre los senos paranasales utilizando un medio de contraste.

\section{Resultados}

\section{Senos Frontales}

El hueso frontal, localizado en la parte rostral superficial y medial de la cabeza, caudal a la cavidad nasal, se utilizó para ubicar los senos frontales. Los senos frontales derecho e izquierdo estaban separados por un septo tabique óseo medio. El seno frontal fue más grande que el seno maxilar. Cada seno frontal estuvo dividido en medial y lateral por un septo incompleto óseo. El seno frontal fue irregular, presentó laminillas intrasinusales, y se comunicó directamente con la fontanela lagrimal.

\section{Seno frontal, porción medial}

Se encontró limitado medialmente por la sutura frontal, caudalmente por una línea imaginaria a la altura del agujero supraorbitario, rostralmente por el hueso nasal y lateralmente por una línea imaginaria desde el agujero supraorbitario hacia el hueso nasal. Esta línea pasó sobre el tabique incompleto óseo que separó la porción medial de la porción lateral (figuras 1 y 2). La porción medial midió aproximadamente $2.5 \mathrm{~cm}$ de largo por $1.9 \mathrm{~cm}$ de ancho.

\section{Seno frontal, porción lateral}

Estuvo delimitado medialmente por una línea imaginaria del agujero supraorbitario hacia rostral, caudalmente por una línea imaginaria aproximadamente a $0.5 \mathrm{~cm}$ del agujero supraorbitario, rostralmente por la fontanela 


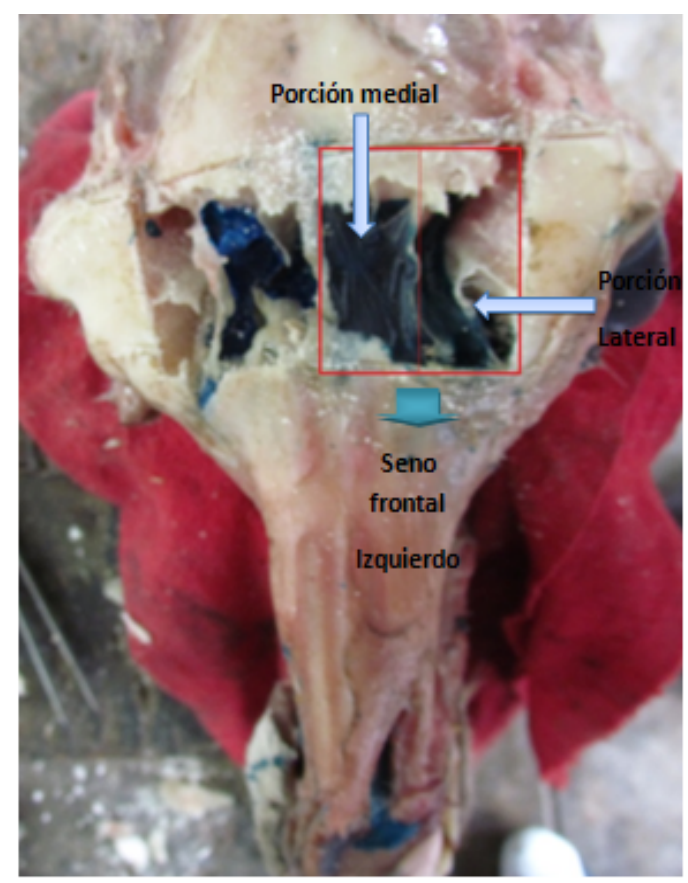

Figura 1. Seno frontal izquierdo de la alpaca, porción medial y lateral lagrimal, y lateramente por una línea imaginaria a la altura del borde supraorbitario. Midió aproximadamente $4 \mathrm{~cm}$ de largo por 1.2 $\mathrm{cm}$ de ancho (figuras 1 y 2 ).

\section{Senos Maxilares}

Los senos maxilares estuvieron localizados en la parte latero-rostral de la cabeza. El seno maxilar ocupó gran parte del hueso maxilar y parte del hueso cigomático, y no presentó compartimentos. No tuvo relación con las raíces de los dientes molares. Midió aproximadamente $2.3 \mathrm{~cm}$ de largo por $1 \mathrm{~cm}$ de ancho (Fig. 3).

El agujero infraorbitario se localizó ventralmente al seno maxilar. Este se comunicó con la cavidad nasal a través de meato nasal ventral por la abertura nasomaxilar. El seno maxilar se limitó rostralmente por una línea imaginaria transversal al agujero

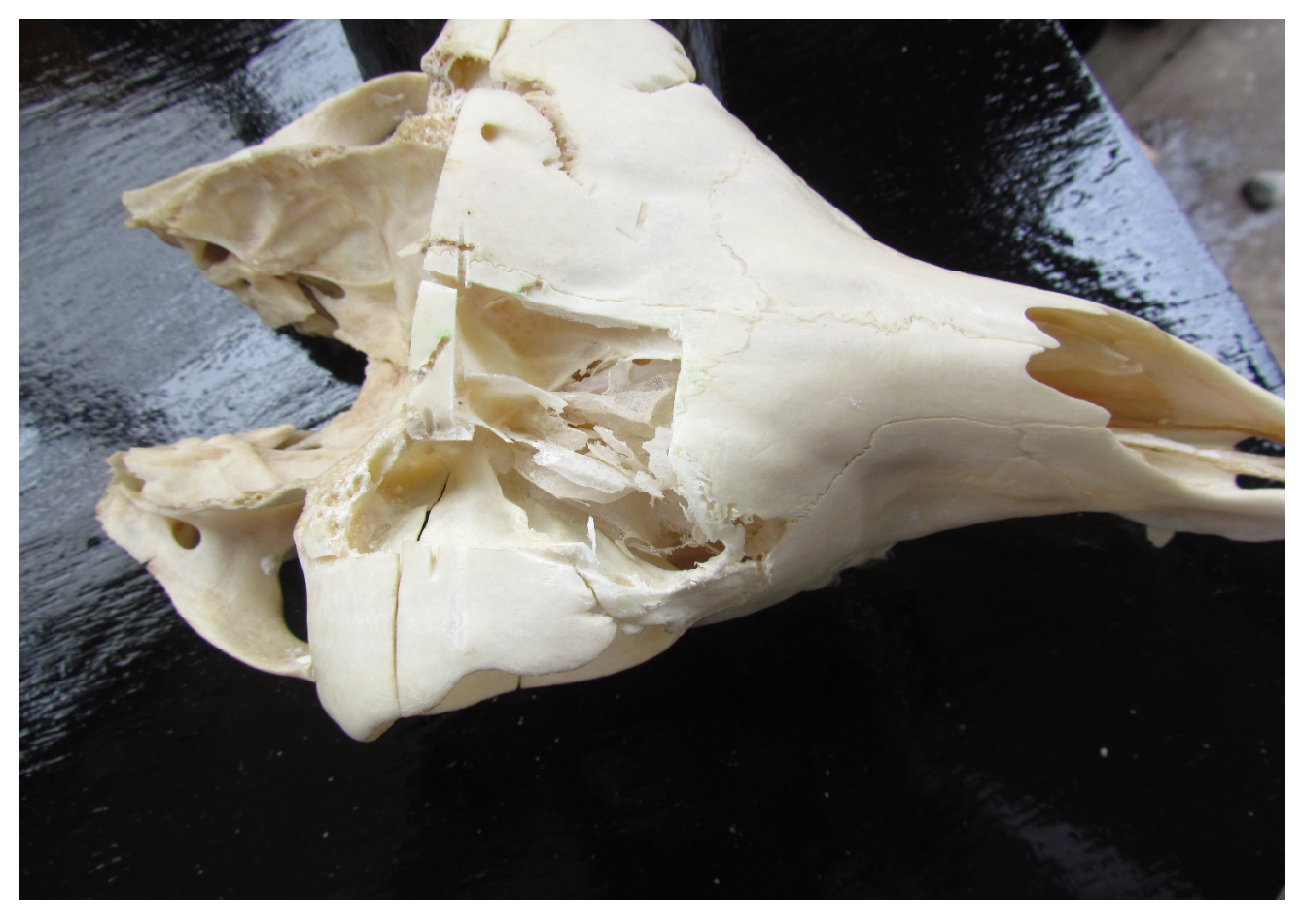

Figura 2. Puntos de referencia de los senos frontales de la alpaca. Límites del seno frontal medial: 1. Línea paralela a la sutura frontal; 2, Altura del agujero supraorbitario; 3. Sutura frontal; 4. Hueso nasal; Límites del seno frontal lateral: 1. Línea paralela a la sutura frontal; 5. Altura de la fontanela lagrimal; 6. Borde supraorbitario; 7. Caudal al agujero supraorbitario $(0.5 \mathrm{~cm})$ 


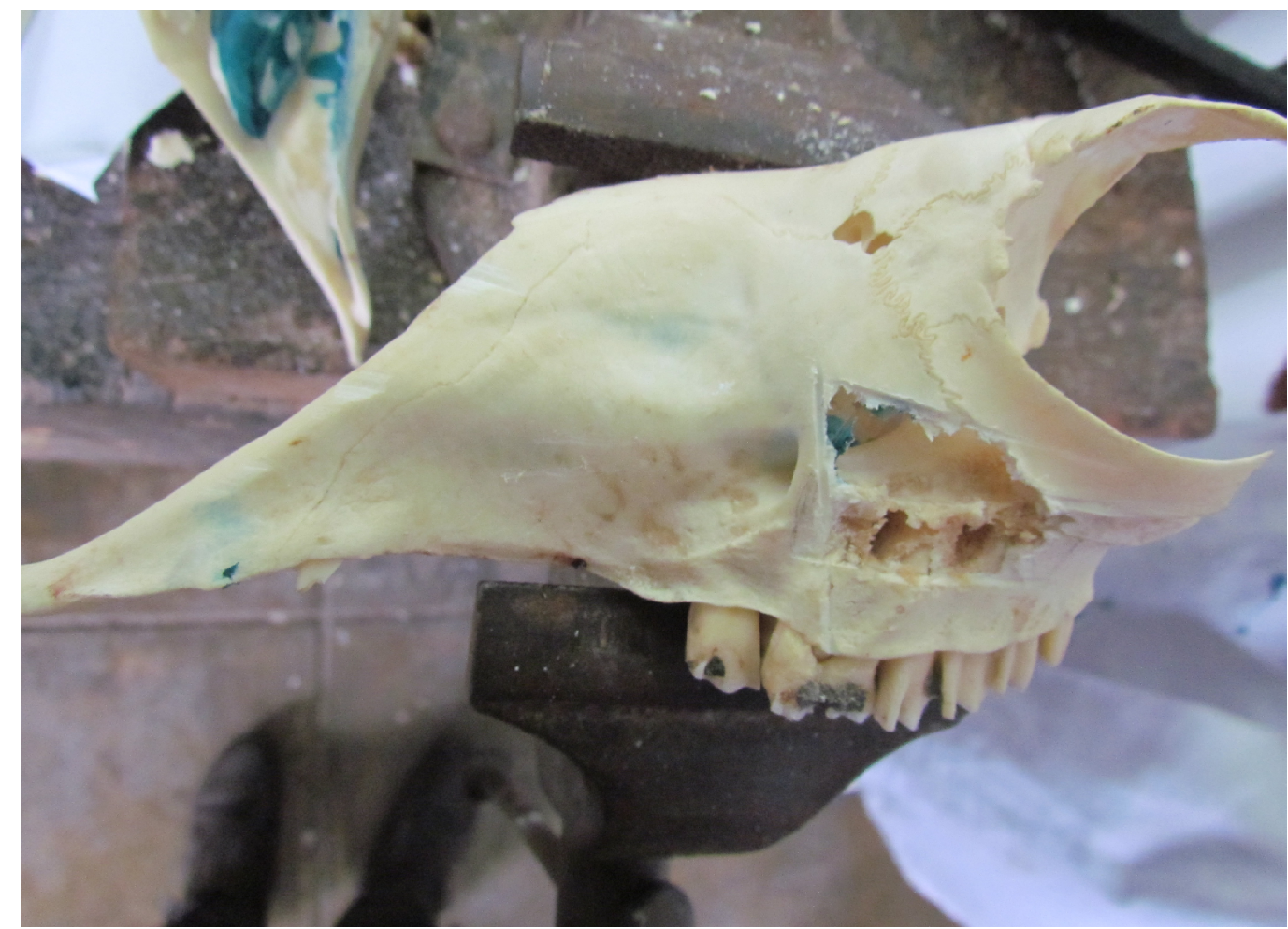

Figura 3. Puntos de referencia del seno maxilar. 1. Rostral, línea transversal al agujero infraorbitario; 2. Ventral, línea imaginaria paralela al agujero infraorbitario; 3. Caudal, borde ventral de la órbita; 4. Dorsal, línea imaginaria a la mitad del hueso cigomático; A. Agujero infraorbotario; B. Hueso cigomático

infraorbitario, ventralmente por una línea imaginaria paralela al agujero infraorbitario, caudalmente con el borde ventral de la órbita y dorsalmente con una línea imaginaria a la mitad del hueso cigomático (Fig. 3).

\section{Radiografía de los Senos Paranasales}

La evaluación de las placas radiográficas fue facilitada por el uso del medio de contraste. En la placa con vista latero-lateral se pudo observar el seno frontal de forma triangular (Fig. 4). El seno maxilar apareció claramente de forma casi rectangular, ubicado dorsalmente a los molares y excavó parte del hueso cigomático (Fig. 5).
Se observó una comunicación entre el seno frontal y el seno maxilar.

En la placa con vista dorso-ventral se observó claramente el seno maxilar, el cual tiene forma rectangular. No se evidenciaron los senos esfenoideos y etmoideos.

\section{Fontanela Lagrimal}

Se observó un foramen delimitado por el seno frontal, el hueso nasal, el hueso lagrimal y el hueso maxilar (Fig. 6). Este foramen tuvo comunicación directa con el seno frontal ya que los dividió un tabique incompleto en la zona central. El foramen estuvo cubierto por una membrana. 


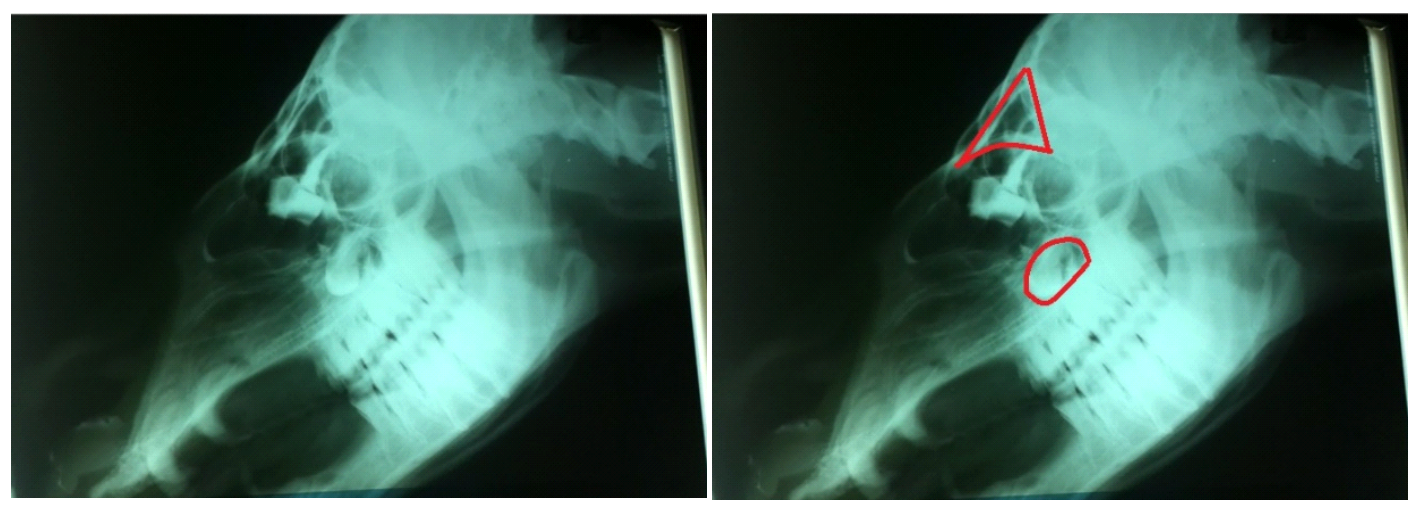

Figura 4. Placas radiográficas con medio de contraste $(5 \mathrm{ml})$. Vista latero-lateral de la cabeza de una alpaca adulta. La vista del lado derecho muestra el seno frontal (triángulo superior) y el seno maxilar (recuadro inferior)

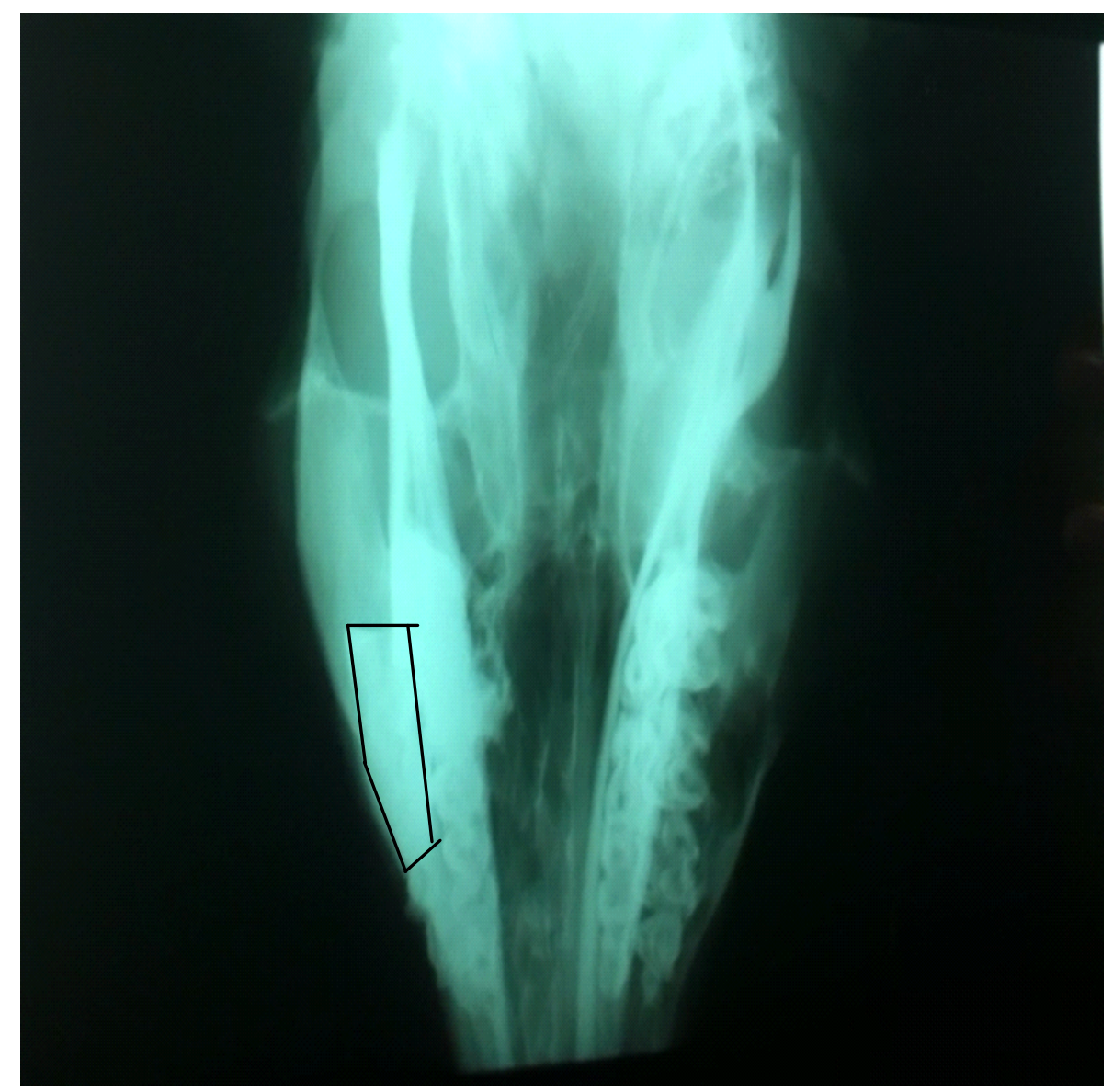

Figura 5. Placa radiográfica con medio de contraste $(5 \mathrm{ml})$ del cráneo de una alpaca, vista dorso-ventral, donde se observa el seno maxilar (recuadro) 


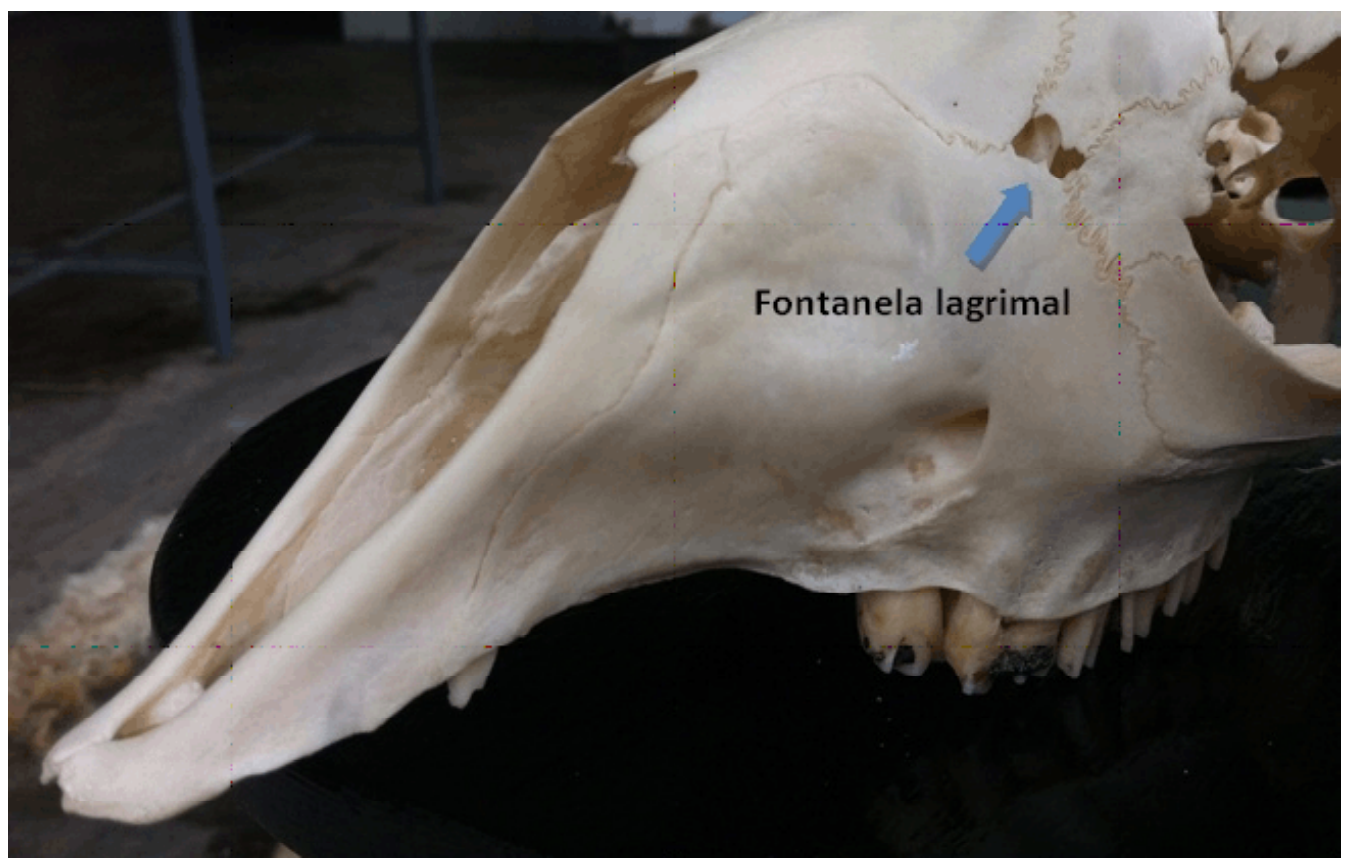

Figura 6. Cráneo de la alpaca mostrando la fontanela lagrimal (flecha)

\section{Discusión}

El seno paranasal frontal se encontró presente como en todos los mamíferos domésticos. En el caballo, pequeños rumiantes y carnívoros está localizado en la parte dorsal de la cabeza entre la cavidad nasal craneal y la órbita (Shively, 1993). Una disposición similar fue encontrada en la alpaca. Si bien es grande, en el bovino es relativamente más grande, ya que en ellos, la arcada cigomática forma una tuberosidad que se continua hasta el cuerno del animal (Sarmiento, 2011).

Los senos frontales de la alpaca presentaron una disposición morfológica semejante al de los rumiantes menores, ya que cuenta con divertículos que lo dividen en seno frontal medial y lateral (Níquel et al., 1973). A su vez, se diferenció del seno frontal del equino, porque en este es indiviso (Shively, 1993).
La comunicación de los senos frontales con la cavidad nasal se asemejó a la del equino, ya que no tiene una comunicación directa sino una comunicación indirecta a través del seno maxilar (Shively, 1993; König y Liebich, 2004). El seno frontal se comunicó con el seno maxilar por la abertura frontomaxilar como en el caso de los equinos (Shively, 1993; König y Liebich, 2004; Climent et al., 2005).

El seno maxilar no albergó las últimas molares superiores, según la fórmula dentaria dada por Wheeler (1982). En el caso del equino, el seno maxilar alberga a los últimos molares (Shively, 1993; Climent et al., 2005). Asimismo, fue indiviso como en el bovino y rumiantes menores; y se diferenció del equino, quien presenta seno maxilar rostral y caudal (Shively, 1993).

Los senos paranasales de la alpaca se diferenciaron de los senos paranasales del camello de una joroba, ya que en estos se presenta un sistema dorsal (seno frontal y 
cornete dorsal) y un sistema ventral (senos maxilares, esfenoidales y etmoidales) (Ahmed et al., 1985), no observados en la alpaca.

\section{Conclusiones}

- El seno frontal se divide en seno frontal lateral y seno frontal medial, similar a los rumiantes menores; y tiene comunicación con los senos maxilares a través del agujero frontomaxilar.

- La fontanela lagrimal tiene comunicación directa con el seno paranasal frontal.

- Los senos frontales son más amplios que los senos maxilares.

- Los senos paranasales maxilares tienen forma irregular y son indivisos como en los rumiantes, pero tienen comunicación con la cavidad nasal a través de la abertura nasomaxilar, como en el equino.

- No se evidenció la presencia de senos paranasales esfenoidal ni etmoidal.

\section{Literatura Citada}

1. Ahmed A, Shokry M, El-Keiey M. 1985. Contribution to the paranasal sinuses of the one humped camel (Camelus dromedarius). Anat Histol Embryol 14:221-225. doi: 10.1111/j.14390264.1985.tb00279.x

2. Ameghino E, DeMartini J. 1991. Mortalidad en crías de alpacas. Bol IVITAUNMSM. p 71-80.

3. Aspinall V, O'Reilly. 2004. Introducción a la anatomía y fisiología veterinarias. Zaragoza, España: Acribia. 255 p.
4. Climent S, Sarasa M, Muniesa P, Latorre R. 2005. Manual de anatomía y embriología de los animales domésticos. Zaragoza, España: Acribia. 423 p.

5. Fowler M. 2010. Medicine and surgery of camelids. $3^{\text {rd }}$ ed. Iowa, USA: WileyBlackwell. 617 p.

6. Iñiguez LC, Alem R. 1996. Role of camelids as means of transportation and exchange in the Andean region of Bolivia. World Anim Rev 86: 12-21.

7. König H, Liebich H. 2004. Anatomía de los animales domésticos. Tomo I. $2^{\text {a }}$ ed. Madrid: Ed Médica Panamericana. $288 \mathrm{p}$.

8. Navarrete M, Sato A. 2010. Aspectos anatómicos de la cría de alpaca. En: Sanidad de alpacas en la etapa neonatal. Manual para estudiantes y profesionales de veterinaria. España: Complutense. p 51-62.

9. Níquel R, Schummer A, Seiferle E. 1973. The viscera of the domestic mammals. $2^{\text {nd }}$ ed. Germany: Verlag Paul Parey. 274 p.

10. Nómina Anatómica Veterinaria. 2012. $5^{\text {th }}$ ed. Hannover, Germany: International Committee on Veterinary Gross Anatomical Nomenclature (ICVGAN). $177 \mathrm{p}$.

11. Sarmiento R. 2011. Semiología clínica veterinaria. Bogotá, Colombia: UDCA. $540 \mathrm{p}$.

12. Shively MJ. 1993. Anatomía veterinaria: básica comparativa y clínica. México DF: El Manual Moderno. 379 p.

13. Sisson S, Grossman JD. 1993. Anatomía de los animales domésticos. $5^{\mathrm{a}}$ ed. Tomo I. España: Salvat Editores. p 209-256.

14. Wheeler JC. 1982. Aging llamas and alpacas by their teeth. Llama World 1 : 12-17. 